\title{
Special chaotic systems
}

\author{
Sajad Jafari ${ }^{1,2, a}$ and Tomasz Kapitaniak ${ }^{3}$ \\ ${ }^{1}$ Health Technology Research Institute, Amirkabir University of Technology, \\ 424 Hafez Ave., Tehran 15875-4413, Iran \\ ${ }^{2}$ Biomedical Engineering Department, Amirkabir University of Technology, \\ Tehran 15875-4413, Iran \\ ${ }^{3}$ Division of Dynamics, Lodz University of Technology, Stefanowskiego 1/15, \\ 90-924 Lodz, Poland
}

Received 4 February 2020

Published online 26 March 2020

\begin{abstract}
This topical issue collects contributions related to recent achievements and scientific progress in special chaotic systems. The individual papers focus on various questions of present-day interest in this topic.
\end{abstract}

For many years, researchers have believed that the formation of strange attractors in a dynamical system is related to a saddle point in its structure. Many well-known systems with chaotic attractors such as the Lorenz, Rossler, Chua, and Chen systems have a saddle point equilibrium. This category of systems with chaotic attractors is familiar to researchers, and finding the chaoti atractors is simple because they are are formed near the saddle points. In other words, most chaotic systems have a strange attractor around their saddle point equilibrium and can be easily designed [1]. In 2011, Sprott presented standards to propose new systems with strange attractors [2]. He proposed that a new chaotic system should satisfy at least one of the following three conditions: first, the proposed systems should model some essential unsolved problems in nature; second, the systems should exhibit some previously unobserved behavior; and finally, the system should be simpler than all other known examples exhibiting the observed behavior. For example, the Lorenz system satisfied all of these conditions in its first publication in 1963.

In the past decade, some novel dynamical systems with chaotic attractors have been found that did not have a saddle point equilibrium [3-8]. To date, many new chaotic systems have been proposed in this category. We call these systems "special". In other words, chaotic systems that satisfy the novelty conditions, and are not common, are in this category. Chaotic systems without any equilibria [9], chaotic systems with a line of equilibria [10], chaotic systems with a curve of equilibria [11], and with surfaces of equilibria $[11,12]$ are in this category.

The dynamics of chaotic systems depend on the initial conditions as well as parameters. So, a system can show different coexisting attractors in the constant parameters just by varying initial conditions [14-16]. Such a system is called "multi-stable" $[17,18]$. A system that has a countable infinity of coexisting attractors

a e-mail: sajadjafari83@gmail.com 
is called "megastable" [19-22], while a system with an uncountable infinity of coexisting attractors is called "extreme multi-stable" [23-25]. Chaotic systems with different multi-stabilities can satisfy the novelty conditions of the standard for proposing chaotic systems. Another interesting dynamic in special chaotic systems is the coexistence of symmetric [26-28] attractors.

Chaotic attractors can be categorized into self-excited or hidden attractors $[1,29]$. Self-excited attractors are those attractors in which their basin of attraction contains an unstable equilibrium, while the basin of attraction in hidden attractors is not related to any equilibrium point [30-33]. Hidden chaotic attractors are one of the hottest topics in the study of special chaotic systems. Many novel systems have been proposed with hidden attractors [34-36]. Chaotic systems with rare attractors have attracted attention. Rare attractors are those attractors in which their basins of attraction are tiny [14,37]. Systems with multi-scroll chaotic attractors are other interesting dynamical systems [38-40].

Chaotic systems can be categorized based on their dissipation. A system is called conservative if its dissipation is zero. Some systems are "nonuniformly conservative" [41]. This means that they are globally conservative, but they have some regions of state space in which the system is dissipative and some other regions that are antidissipative. Also, there are certain other features that are important to consider in the study of new chaotic systems.

This special issue reviews the current state of the art in the research on special chaotic systems. It starts with two mini-reviews. The author in paper [42] reviews fractional-order chaotic systems. It discusses the history, achievements, applications, and future challenges in fractional-order chaotic systems. The review begins with a brief history of the first publications on fractional-order chaotic systems. Then, it continues by investigating recent relevant progress. A summary of particular applications for such systems, which have been reported in the literature, is presented. The paper is closed by discussing some open problems on this research subject. Authors in [43] give a comprehensive review of chaotic flows with special equilibria. In their review, they categorize chaotic systems with special properties in their equilibrium into eight groups: systems with no equilibrium (NE), with stable equilibrium (SE), with line equilibrium (LE), with curve equilibrium (CE), with plane equilibrium (PE), with surface equilibrium (ES), with unstable equilibrium (US), and systems that belong to more than one category (chameleon systems). They introduce the pioneer works of each group and also review some recent important papers related to these groups.

Some of the papers investigate chaotic systems from the real-world [44-51]. Authors in [48] discuss a chaotic map of the process equation as a model for the development of cells. They propose a behavioral model for cells that shows different dynamics, from a high pluripotent stem cell to any distinct cell fate. The proposed model considers a cell as a black-box for a living system and tries to depict the presumed behaviors of the system. The model is a multi-stable iterated map with a sensitive dependence on initial conditions. In [51], the authors propose an extended Hindmarsh-Rose neuron model by taking into consideration slowly interacting cell phenomena due to calcium ions. In the extended model, they consider the effect of an external forcing current and the electromagnetic coupling between the magnetic flux and the membrane potential of the neuron. They analyze the modified neuron model in the presence of periodic and quasi-periodic excitations. The results demonstrate multi-stability and hyperchaos, which was not explored earlier. To model diabetes mellitus pathophysiology, authors in [44] propose a computational model for the insulin-glucose regulatory system. In this differential equation model, the complex behavior of this biological system is considered. This model shows chaos and bifurcating properties, which have been observed in dynamical diseases. The authors have analyzed the static and dynamical properties of the proposed model to show its 
strength and capability to represent different types of diabetes and other dysfunctions in the insulin-glucose system. Another research study related to the neuronal system is [47]. In this work, the authors study a four-dimensional inertial two-nervous system with delay. By analyzing the distribution of eigenvalues, the critical value of zero-Hopf bifurcation is obtained. Complex dynamic behaviors are considered when two parameters change simultaneously. Pitchfork and Hopf bifurcation critical lines near the zero-Hopf point are obtained by using the central manifold reduction and normal form theory. The bifurcation diagram is given, and the results of perioddoubling bifurcation into the chaotic region in the inertial two-neural system with delayed Crespi function are shown. Alongside biological systems, other papers are about specific real-world phenomena. The Nose-Hoover oscillator is a well-studied chaotic system originally proposed to model a harmonic oscillator in equilibrium with a heat bath at a constant temperature. Although this is a simple three-dimensional system with five terms and two quadratic nonlinearities, it displays a wide variety of unusual dynamics. However, it falls considerably short of its original purpose. The author in [50] describes two simple variants of the Nose-Hoover oscillator, the first of which satisfies the original goal exactly, and the second of which exhibits a hidden global chaotic attractor that fills all of its three-dimensional state space. The author in [45] states that during the last six years or so, several exciting papers have discussed systems with line segments of equilibria or planes of equilibria, and systems with more general configurations. It draws attention to the fact that such equilibria were considered previously by Miklos Farkas (1932-2007), in papers published in 1984-2005. He called zip bifurcations those involving line segments of equilibria, and velcro bifurcations those involving planes of equilibria. Then the author briefly describes situations involving zip and velcro bifurcations. Authors in [49] propose dynamical system governing from the nonlinear extension of the electrostatic ionacoustic waves (IAWs) under the influence of the external magnetic field in ThomasFermi (TM) consisting of hot electrons, cold electrons, and mobile ions. The coupled lower-dimensional model with a planar Hamiltonian exhibits periodic oscillations and conservative properties. The disrupted system with trigonometric forcing can produce richer phenomena. Authors in [46] study the dynamics of indirect field-oriented control (IFOC) of 3-phase induction motors. The dynamical behaviors of the studied system are investigated using bifurcation diagrams, maximum Lyapunov exponent plots, phase portraits, and isospike diagrams. The numerical simulation results reveal that the IFOC of 3-phase induction motors displays coexistence of attractors for the same set of IFOC of 3-phase induction motor parameters, i.e. periodic and chaotic bursting oscillations. Basins of attraction of different competing attractors are plotted, showing complex basin boundaries.

The next five papers investigate chaotic circuits [52-56]. By introducing a memristor into a chaotic system with a single non-quadratic term and substituting an absolute value function for conditional symmetry, a unique chaotic system is constructed in [52]. The system shares a special structure of symmetry and conditional symmetry. Also, the amplitude and frequency of the system variables can be rescaled by the applied memristor. Interestingly, the system illustrates a new case of attractor control, namely partial amplitude control and global frequency control. Lastly, as a new regime of extreme multi-stability, the memristive system shows relatively simple bifurcation according to the initial condition. Paper [56] demonstrates numerically and experimentally observed transitions between the stable states: regular and chaotic oscillations in the case of state representations of binary memory transformed into the Jordan form. The math model of original memory describes a simple anti-series connection of two resonant tunneling diodes (RTDs). A derived thirdorder dynamical system is analyzed concerning global behavior and, consequently, implemented as a lumped analog circuit with a piecewise linear (PWL) vector field. 
Oscilloscope screenshots prove that the chaotic motion of binary memory is robust. Authors in [53] study extreme and critical events in the forced Lienard systems with a charge control memristor. It has been found that the system exhibits hidden attractors either in the absence or presence of an external sinusoidal force. They give evidence that these attractors play a crucial role in the appearance of critical events. They explain the mechanism leading to the emergence of catastrophic transitions. Finally, they show that the observed critical transitions are typical for memristor-based models, and understanding of them gives some insight into how to avoid these types of devastating events at the time of the device fabrication process. Paper [54] generalizes a second-order memristor-based jerk circuit. This is achieved by substituting the first-order memristor contained diode-bridge and RC filter in an existing memristive jerk circuit with a second-order one composed of a diode-bridge and LC network. The second-order-memristor-based jerk circuit possesses an unstable saddle-focus and generates complex parameter-dependent dynamics, including hyperchaos, chaos, quasi-period, and period along with co-existing behaviors. The coexistence of symmetric chaotic and quasi-periodic attractors is shown by local attraction basins. Furthermore, hardware breadboard is made for experimental investigations, and the measurement results strongly validate complex parameter-dependent dynamics revealed by the numerical simulations. In [55], conditional symmetry is obtained in a memristive system when the function-based polarity inverse meets the new polarity balance, which produces coexisting oscillations, including chaos and other periodic ones. Coexisting bifurcations in two separate spaces are studied, showing an interesting function of amplitude modification in a limited parameter interval. Furthermore, a constant is embedded in the system as a knob to control the coexisting solutions with any desired offset in one dimension. A circuit is designed showing the same dynamics as numerical simulation.

While most papers in this special issue are about chaotic flows, some works about chaotic maps are presented [57-60]. In [58], the authors study relatively simple examples of non-autonomous maps having different changes in time chaotic attractors. They present a definition for a non-stationary hyperbolic attractor of the driven maps and prove the existence of a non-stationary hyperbolic attractor in a 2-D driven map. Also, a hyperchaotic attractor is introduced for the autonomous 3-D map of the master-slave structure. Paper [59] studies the dynamics of a new fractional-order map with no fixed points. Through phase plots, bifurcation diagrams, and the largest Lyapunov exponent, it is shown that the proposed fractional map exhibits chaotic and periodic behavior. New Hidden chaotic attractors are observed, and the transient state is found to exist. The complexity of the new map is also analyzed by employing approximate entropy. Similarly, a control scheme is introduced. The controllers stabilize the states of the fractional map and ensure their convergence to zero asymptotically. Authors in [57], propose a new model of chaotic attractors. The parameters of the proposed model are varied like the state variables of the traditional chaotic attractors. The variation ranges and values of the varied parameters are designed to produce the required chaotic attractors. As the parameter variation of the chaotic systems affects the chaotic attractors, it also affects the Lyapunov exponents and the complexity of the chaotic systems. The results of the numerical simulation show that the variation process of the parameters can positively affect the sensitivity of the system to its initial conditions, which increase the values of the largest Lyapunov exponent. In [60], the authors introduce a dynamical map with an infinite number of equilibrium points. Although chaotic flows with special properties in their equilibria have been investigated widely, this kind of work on chaotic maps has been neglected. A complete dynamical analysis is done on this new map, which reveals its productive potential in showing chaos and multistability. 
Multi-stability is undoubtedly a very hot topic in nonlinear dynamics. In this issue, many papers [61-66] deal with multi-stability. In [63], the authors study the dynamical behavior of an optomechanical system. It is shown that this optomechanical system can exhibit antimonotonicity, coexisting attractors, and periodic and chaotic bubble behaviors for a specific choice of the parameters. In the resolved sideband regime and when the time scale of the mechanical oscillations is sufficiently larger than the time scale of the cavity field, this optomechanical system displays Hopf bifurcation, which triggers bursting oscillations. Paper [64] investigates the dynamics of a model of a 4-neuron-based hyperchaotic Hopfield neural network (HHNN) with a unique unstable node as a fixed point. The basic properties of the model, including symmetry, dissipation, and condition of the existence of an attractor, are explored. The numerical simulations highlight several complex phenomena such as periodic orbits, quasi-periodic orbits, and chaotic and hyperchaotic orbits. Interestingly, the authors describe several sets of synaptic weight matrixes for which the HHNN studied displays multiple coexisting attractors, including two, three, and four symmetric and disconnected attractors. In [61], two simple megastable systems are presented in detail. These systems show hyperchaotic behavior, which is a property in these types of systems as previously proposed systems mostly show chaos and periodic responses. In [66], a fourth-order hyperchaotic circuit with smooth diode-based nonlinearity is modified from the Tamasevicius oscillator. The hyperchaotic circuit has both simple topology and algebraic equations. Certain previously uncovered dynamical behaviors of multiple coexisting bifurcations, bi-stability, and offset boosting are revealed by theoretical analyses and numerical simulations. In [62], a new 5D chaotic system with line equilibrium is designed and described to reveal its extreme multistability. All of the resulting attractors are hidden. The suggested system has many complex dynamic behaviors in comparison with other chaotic systems. Also, the line equilibrium stability in detail, bifurcation diagrams, Lyapunov exponents, and basins of attraction are demonstrated. In [65], an autonomous RC hyperjerklike chaotic circuit with cubic nonlinearity is introduced and investigated. The state equations of the proposed hyperjerk chaotic circuit are described using Kirchhoff's laws. A number of fundamental properties of the system, such as symmetry, dissipation, equilibrium points, and stability are examined. By varying the parameters of the system, it is revealed from numerical simulations that the system exhibits some interesting dynamics, including crisis events, hysteretic dynamics (inducing the coexistence of attractors), and transient chaos.

Some of the papers focus on chaotic systems with hidden attractors [67-69]. In [69], an integer- and fractional-order form of a four-dimensional (4-D) chaotic system with hidden attractors is investigated using theoretical and numerical methods. The system is constructed by modifying the well-known two-dimensional Lotka-Volterra system. When the selected bifurcation parameter varied, the system exhibits various dynamical behaviors and features, including the intermittency route to chaos, chaotic bursting oscillations, and offset boosting. Moreover, the fractional-order form of the system is examined through bifurcation analysis. In [67], a 3-D multi-statetime-delayed chaotic system consisting of an externally added memristive element is proposed. The proposed system belongs to the family of hidden attractors and displays multi-scroll behaviors. The chaotic behavior of the proposed time-delayed system is investigated by bifurcation analysis, phase-plane analysis, and instantaneous phase plot. Finally, a sliding mode controller is proposed for switching synchronization between the two identical proposed multi-state-time-delayed chaotic systems, including an externally added memristor with disturbances. Authors in [68] study an on-off feedback method to control the dynamics of a radio physical oscillator having hidden coexisting attractors. The proposed time-varying on-off feedback effectively controls the multi-stability, which acts in an on-off manner. They show that for 
suitable values of feedback strength, feedback active time ratio, and time period, the coexisting chaotic and periodic dynamics of the system go to a mono-stable periodic state, followed by a stable steady state.

Several interesting chaotic systems have special features related to their equilibria [70-76]. In [70], a new chaotic system in the spherical coordinates is proposed. The system's solution is inside a predefined sphere, and its attractor cannot cross the sphere. Investigation of equilibrium points of the system shows that the system has eight equilibria, and all of them are saddle. Bifurcation analysis of the system depicts the period-doubling route to chaos with changing the bifurcation parameter. The basin of attraction of the system is investigated to show the sensitivity of the system to initial conditions. In [75], a three-dimensional novel chaotic system and its projective synchronization are investigated. The proposed chaotic system has no equilibria. The topological structure of the proposed chaotic system is different from Lorenz, Rossler and Chen systems. Different qualitative and quantitative tools such as time series, phase plane, Poincare section, bifurcation plot, Lyapunov exponents, Lyapunov spectrum, and Lyapunov dimension are used to demonstrate the chaotic behavior of the proposed system. Further, the projective synchronization between the proposed chaotic systems is achieved using nonlinear active control. In [73], a new hyperchaotic memristor oscillator is proposed. Different dynamical properties of the proposed system, such as dissipativity, equilibrium points, and their stabilities, Lyapunov exponents and Kaplan-Yorke dimension are investigated. The system has a line of equilibria. Therefore, it belongs to the category of systems with hidden attractors. Investigation of the stability of the line of equilibria shows that the line is stable in some intervals and unstable in others. Bifurcation analysis of the system reveals several coexisting attractors in certain ranges of parameters, which indicates multi-stability. Authors in [74] propose the simplest three-dimensional chaotic flow that has a line of equilibria. The chaotic attractor of the system is very special with two slow and fast parts. In other words, the dynamics of the system are a combination of slow and fast states. The unique chaotic attractor of the system is investigated. Dynamical properties of the system, such as stability of equilibrium points and bifurcation diagrams, are studied. Authors in [71] propose a novel autonomous threedimensional system exhibiting a hidden attractor. Its attractor cannot be tracked using perpetual points. The reason behind this inefficiency is explained using the theory of differential equations. This system consists of a slow manifold depicted through the time-series, although the system has no equilibrium points or such multiplicative parameters. The authors also discuss the behavior of the attractor using timeseries analysis, bifurcation theory, Lyapunov spectrum, and Kaplan-Yorke dimension. Authors in [72] report a class of systems without equilibria, which exhibit a scroll attractor and whose vector field is differentiable. The system construction presents great flexibility for the selection of the number of scrolls exhibited by the attractor. They also report a special coupling for this class of systems, which allows the coupling without introducing new equilibria in the system. In paper [76], a 3D jerk system with only one stable equilibrium and hidden attractor is analyzed in infinity with the help of the Poincare compactification. Meanwhile, a distributed delayed feedback (DDF) control scheme for this system is proposed. By using the center manifold theory of functional differential equation (FDE), Hopf bifurcation for the DDF control system is analyzed and obtained. Results confirm the accuracy of the bifurcation analysis and the effectiveness of the proposed DDF control strategy

Finally, the last four papers are related to special chaotic flows, such as conservative, multi-scroll systems, and so on. Based on the matrix differential equation of the Sprott-A system, paper [77] presents a class of rare 3D conservative systems by adjusting its skew-symmetric state matrix and Hamiltonian. Then, an example system is reported to show conservative dynamical behaviors. For given parameters and 
initial conditions, the example system can generate six isolated invariant tori and six cluster-conservative chaotic flows. In [78], a new three-dimensional continuous autonomous system is proposed. It exhibits single scroll chaotic behavior in a particular parameter region. By linear stability analysis and numerical simulations, the authors investigate different dynamical observations with respect to system parameters and try to understand the route of generation of chaos. Lyapunov exponent and Kaplan-Yorke dimension are used to verify the chaotic behavior. It becomes periodic via an inverse period-doubling route for higher values of the parameters. A two-parameter bifurcation diagram is shown, which distinguishes the chaotic region from other periodic and steady-state regions. In [79], the authors introduce a chaotic square attractor based on the collision of two heteroclinic orbits. Before the collision, the system presents the coexistence of two double scroll attractors. They are generated via piecewise linear (PWL) systems that deal with two saddle-foci equilibria of different classes. In [80], a new function is introduced to generate various multi-double-scroll and multi-double-wing hidden attractors. In order to prove the effectiveness of the proposed method, three chaotic systems are taken into consideration: a 3D chaotic system with stable equilibria, a $4 \mathrm{D}$ chaotic system, and a 4D hyper-chaotic system, both without equilibrium. The dynamical behaviors of these systems are analyzed theoretically and numerically simulated, such as analysis of equilibria and their stability, Lyapunov exponent spectra, and bifurcation diagrams.

This special issue provides a broad spectrum of current research on special chaotic systems, and we hope that the researchers who are active in this field will find it useful. We wish to express our appreciation to the authors of all the papers in this special issue for their excellent contributions, as well as the many reviewers for their high-quality work on reviewing the manuscripts.

\section{References}

1. D. Dudkowski, S. Jafari, T. Kapitaniak, N.V. Kuznetsov, G.A. Leonov, A. Prasad, Phys. Rep. 637, 1 (2016)

2. J.C. Sprott, Int. J. Bifurc. Chaos 21, 2391 (2011)

3. Z. Wei, Phys. Lett. A 376, 102 (2011)

4. X. Wang, G. Chen, Commun. Nonlinear Sci. Numer. Simul. 17, 1264 (2012)

5. T. Gotthans, J. Petržela, Nonlinear Dyn. 81, 1143 (2015)

6. S. Jafari, J.C. Sprott, Chaos Solitons Fractals 57, 79 (2013)

7. S. Jafari, J.C. Sprott, S.M.R. Hashemi Golpayegani, Phys. Lett. A. 377, 699 (2013)

8. M. Molaie, S. Jafari, J.C. Sprott, S.M.R. Hashemi Golpayegani, Int. J. Bifurc. Chaos 23, 1350188 (2013)

9. S. Ren, S. Panahi, K. Rajagopal, A. Akgul, V.-T. Pham, S. Jafari, Z. Naturforsch. A. 73, 239 (2018)

10. V.T. Pham, S. Jafari, C. Volos, L. Fortuna, Chaos Solitons Fractals 120, 213 (2019)

11. K. Barati, S. Jafari, J.C. Sprott, V.-T. Pham, Int. J. Bifurc. Chaos 26, 1630034 (2016)

12. S. Jafari, J.C. Sprott, M. Molaie, Int. J. Bifurc. Chaos 26, 1650098 (2016)

13. S. Jafari, J.C. Sprott, V.-T. Pham, C. Volos, C. Li, Nonlinear Dyn. 86, 1349 (2016)

14. A. Chudzik, P. Perlikowski, A. Stefanski, T. Kapitaniak, Int. J. Bifurc. Chaos 21, 1907 (2011)

15. P. Jaros, P. Perlikowski, T. Kapitaniak, Eur. Phys. J. Special Topics 224, 1541 (2015)

16. T. Kapitaniak, G.A. Leonov, Eur. Phys. J. Special Topics 224, 1405 (2015)

17. A.N. Pisarchik, U. Feudel, Phys. Rep. 540, 167 (2014) 
18. Z. Wei, V.-T. Pham, A.J.M. Khalaf, J. Kengne, S. Jafari, Int. J. Bifurc. Chaos 28, $1850085(2018)$

19. Y.-X. Tang, A.J.M. Khalaf, K. Rajagopal, V.-T. Pham, S. Jafari, Y. Tian, Chin. Phys. B. 27, $40502(2018)$

20. S. Jafari, K. Rajagopal, T. Hayat, A. Alsaedi, V.-T. Pham, Int. J. Bifurc. Chaos 29, 1950187 (2019)

21. Z. Wang, I.I. Hamarash, P.S. Shabestari, S. Jafari, Int. J. Bifurc. Chaos 29, 1950176 (2019)

22. J.C. Sprott, S. Jafari, A.J.M. Khalaf, T. Kapitaniak, Eur. Phys. J. Special Topics 226, 1979 (2017)

23. C. Hens, R. Banerjee, U. Feudel, S. Dana, Phys. Rev. E 85, 035202 (2012)

24. D.A. Prousalis, C.K. Volos, B. Bao, E. Meletlidou, I.N. Stouboulos, I.M. Kyprianidis, Extreme multistability in a hyperjerk memristive system with hidden attractors, in Recent Advances in Chaotic Systems and Synchronization (Elsevier, 2019), p. 89-103

25. Y. Zhang, Z. Liu, H. Wu, S. Chen, B. Bao, Chaos Solitons Fractals 127, 354 (2019)

26. J.C. Sprott, Int. J. Bifurc. Chaos 25, 1550078 (2015)

27. C. Li, J.C. Sprott, Y. Liu, Z. Gu, J. Zhang, Int. J. Bifurc. Chaos 28, 1850163 (2018)

28. C. Li, Y. Xu, G. Chen, Y. Liu, J. Zheng, Nonlinear Dyn. 95, 1245 (2019)

29. D. Dudkowski, A. Prasad, T. Kapitaniak, Phys. Lett. A 379, 2591 (2015)

30. N. Kuznetsov, G. Leonov, M. Yuldashev, R. Yuldashev, Commun. Nonlinear Sci. Numer. Simul. 51, 39 (2017)

31. N. Kuznetsov, G. Leonov, T. Mokaev, A. Prasad, M. Shrimali, Nonlinear Dyn. 92, 267 (2018)

32. G. Leonov, N. Kuznetsov, V. Vagaitsev, Phys. Lett. A. 375, 2230 (2011)

33. G. Leonov, N. Kuznetsov, V. Vagaitsev, Physica D 241, 1482 (2012)

34. V.-T. Pham, C. Volos, S. Jafari, T. Kapitaniak, J. Circuits Syst. Comput. 27, 1850066 (2018)

35. K. Rajagopal, F. Nazarimehr, S. Jafari, A. Karthikeyan, Eur. Phys. J. Special Topics 226, 3827 (2017)

36. H.R. Abdolmohammadi, A.J.M. Khalaf, S. Panahi, K. Rajagopal, V.-T. Pham, S. Jafari, Pramana 90, 70 (2018)

37. S. Brezetskyi, D. Dudkowski, T. Kapitaniak, Eur. Phys. J. Special Topics 224, 1459 (2015)

38. E. Tlelo-Cuautle, A. Pano-Azucena, J. Rangel-Magdaleno, V. Carbajal-Gomez, G. Rodriguez-Gomez, Nonlinear Dyn. 85, 2143 (2016)

39. J. Ma, X. Wu, R. Chu, L. Zhang, Nonlinear Dyn. 76, 1951 (2014)

40. Z. Liu, F. Wu, F. Alzahrani, J. Ma, Mod. Phys. Lett. B. 32, 1850399 (2018)

41. J.C. Sprott, Elegant chaos: algebraically simple chaotic flows (World Scientific, 2010)

42. M.S. Tavazoei, Fractional order chaotic systems: history, achievements, applications, and future challenges 229, 887 (2020)

43. Z. Wang, Z. Wei, K. Sun, S. He, H. Wang, Q. Xu et al., Chaotic flows with special equilibria 229, 905 (2020)

44. A.M. Ali, F.R. Tahir, Computational model of insulin-glucose regulatory system to represent type 1 diabetes mellitus, hypoglycemia and hyperinsulinemia 229, 943 (2020)

45. J.A.C. Gallas, Zip and velcro bifurcations in competition models in ecology and economics 229, 973 (2020)

46. A.S. Kemnang Tsafack, J.R. Mboupda Pone, A. Cheukem, R. Kengne, G. Kenne, Coexisting attractors and bursting oscillations in IFOC of 3-phases induction motor 229, 989 (2020)

47. Y. Li, L. Xiao, Z. Wei, W. Zhang, Zero-Hopf bifurcation analysis in an inertial twoneural system with delayed Crespi function 229, 953 (2020)

48. F. Nazarimehr, S.S. Hosseini, A.J.M. Khalaf, S. Jafari, J.C. Sprott, Process equation as a model for the development of cells 229, 921 (2020)

49. A. Saha, S. Sarkar, S. Banerjee, K.K. Mondal, Signature of chaos and multistability in a Thomas-Fermi plasma 229, 979 (2020) 
50. J.C. Sprott, Variants of the Nosé-Hoover oscillator 229, 963 (2020)

51. S. Wang, S. He, K. Rajagopal, A. Karthikeyan, K. Sun, Route to hyperchaos and chimera states in a network of modified Hindmarsh-Rose neuron model with electromagnetic flux and external excitation 229, 929 (2020)

52. Z. Gu, C. Li, X. Pei, C. Tao, Z. Liu, A conditional symmetric memristive system with amplitude and frequency control 229, 1007 (2020)

53. S. Leo Kingston, K. Suresh, K. Thamilmaran, Extreme and critical transition events in the memristor based Lienard system 229, 1033 (2020)

54. F. Li, C. Tai, H. Bao, J. Luo, B. Bao, Hyperchaos, quasi-period and coexisting behaviors in second-order-memristor-based jerk circuit 229, 1045 (2020)

55. T. Lu, C. Li, X. Wang, C. Tao, Z. Liu, A memristive chaotic system with offset-boostable conditional symmetry 229, 1059 (2020)

56. J. Petrzela, Binary memory with orthogonal eigenspaces: from stable states to chaotic oscillations 229, 1021 (2020)

57. A.O.A. Alamodi, K. Sun, Y. Peng, Chaotic attractor with varied parameters 229, 1095 (2020)

58. N.V. Barabash, V.N. Belykh, Chaotic driven maps: Non-stationary hyperbolic attractor and hyperchaos 229, 1071 (2020)

59. A.A. Khennaoui, A. Ouannas, S. Boulaaras, V.-T. Pham, A.T. Azar, A fractional map with hidden attractors: chaos and control 229, 1083 (2020)

60. Z. Wang, A.J.M. Khalaf, H. Tian, A. Alsaedi, T. Hayat, A chaotic map with infinite number of equilibria in a bounded domain 229, 1109 (2020)

61. B. Chen, K. Rajagopal, I.I. Hamarash, A. Karthikeyan, I. Hussain, Simple megastable oscillators with different type of attractors; tori, chaotic and hyperchaotic ones $\mathbf{2 2 9}$, 1155 (2020)

62. A.J.M. Khalaf, H.R. Abdolmohammadi, A. Ahmadi, L. Moysis, C. Volos, I. Hussain, Extreme multi-stability analysis of a novel 5D chaotic system with hidden attractors, line equilibrium, permutation entropy and its secure communication scheme 229, 1175 (2020)

63. S.T. Kingni, C. Tchodimou, D.P. Foulla, P. Djorwe, S.G.N. Engo, Antimonotonicity, coexisting attractors and bursting oscillations in optomechanical system: Analysis and electronic implementation 229, 1117 (2020)

64. Z.T. Njitacke, S.D. Isaac, J. Kengne, A.N. Negou, G.D. Leutcho, Extremely rich dynamics from hyperchaotic Hopfield neural network: Hysteretic dynamics, parallel bifurcation branches, coexistence of multiple stable states and its analog circuit implementation 229, 1133 (2020)

65. V. Kamdoum Tamba, E.R. Feudjio, F. Kapche Tagne, J. Noumbissie Fankam, H.B. Fotsin, Crisis event, hysteretic dynamics inducing coexistence of attractors and transient chaos in an autonomous RC hyperjerk like-chaotic circuit with cubic nonlinearity $\mathbf{2 2 9}$, $1189(2020)$

66. Y. Yang, K. Ren, H. Qian, X. Yao, A simple hyperchaotic circuit with coexisting multiple bifurcations and offset boosting 229, 1163 (2020)

67. N.P. Mohanty, R. Dey, B.K. Roy, Switching synchronisation of a 3-D multi-state-timedelay chaotic system including externally added memristor with hidden attractors and multi-scroll via sliding mode control 229, 1231 (2020)

68. A. Sharma, K. Yadav, M.D. Shrimali, A. Prasad, N.V. Kuznetsov, Time varying feedback control on multi-stability in hidden attractor 229, 1245 (2020)

69. V. Kamdoum Tamba, G.H. Kom, S.T. Kingni, J.R.M. Pone, H.B. Fotsin, Analysis and electronic circuit implementation of an integer- and fractional-order four-dimensional chaotic system with offset boosting and hidden attractors 229, 1211 (2020)

70. L. Chen, E. Tlelo-Cuautle, I.I. Hamarash, V.-T. Pham, H.R. Abdolmohammadi, A novel chaotic system in the spherical coordinates 229, 1257 (2020)

71. S.N. Chowdhury, D. Ghosh, Hidden attractors: A new chaotic system without equilibria 229, 1299 (2020)

72. R.J. Escalante-Gonzalez, E. Campos, Hyperchaotic attractors through coupling of systems without equilibria 229, 1309 (2020) 
73. Y. Feng, K. Rajagopal, A.J.M. Khalaf, F.E. Alsaadi, F.E. Alsaadi, V.-T. Pham, A new hidden attractor hyperchaotic memristor oscillator with a line of equilibria 229, 1279 (2020)

74. F. Nazarimehr, J.C. Sprott, Investigating chaotic attractor of the simplest chaotic system with a line of equilibria 229, 1289 (2020)

75. P.P. Singh, B.K. Roy, A novel chaotic system without equilibria, with parachute and thumb shapes of Poincare map and its projective synchronization 229, 1265 (2020)

76. Z. Wang, X. Xi, L. Kong, Z. Wei, Infinity dynamics and DDF control for a chaotic system with one stable equilibrium 229, 1319 (2020)

77. Y. Li, S. Cang, Z. Kang, Z. Wang, A new conservative system with isolated invariant tori and six-cluster chaotic flows 229, 1335 (2020)

78. M.S. Anwar, G.K. Sar, A. Ray, D. Ghosh, Behavior al study of a new chaotic system 229, 1343 (2020)

79. H.E. Gilardi-Velazquez, R.J. Escalante-Gonzalez, E. Campos, Emergence of a square chaotic attractor through the collision of hetero clinic orbits 229, 1351 (2020)

80. Q. Xie, Y. Zeng, Generating different types of multi-double-scroll and multi-double-wing hidden attractors 229, 1361 (2020) 